\title{
El procedimiento legislativo ordinario en la práctica: los acuerdos en primera lectura*
}

\author{
The Ordinary Legislative Procedure in Practice: \\ First Reading Agreements \\ María Gómez-Leal Pérez** \\ Letrada del Parlamento Europeo
}

\begin{abstract}
Sumario: I. Introducción.-II. La evolución de la codecisión: los acuerdos en primera lectura.-III. La apertura de las negociaciones: el mandato de negociación.-IV. Las negociaciones entre Parlamento y Consejo; el papel de la Comisión: los trílogos.-V. La formalización del acuerdo.-VI. Conclusiones.
\end{abstract}

Resumen: El artículo analiza el funcionamiento del procedimiento legislativo ordinario (PLO) en la práctica y cómo la práctica de los «trílogos» y los acuerdos en primera lectura está cada vez más consolidada. La descripción del PLO del artículo 294 TFUE no permite, por sí sola, hacerse una idea exacta del funcionamiento de la PLO en la práctica. Es necesario tener presente la Declaración Común del Parlamento, del Consejo y de la Comisión sobre las modalidades prácticas del procedimiento de codecisión. Como consecuencia de estas modalidades prácticas el número de actos legislativos adoptados en primera lectura está en constante aumento, mientras que el número de propuestas legislativas que llegan a la fase de conciliación se ha reducido considerablemente. El papel del ponente y de la comisión parlamentaria competente así como el de la Presidencia semestral del Consejo se han visto reforzados, en la medida en que asumen el papel de negociadores de sus respectivas instituciones.

Palabras clave: Procedimiento legislativo ordinario (PLO), instituciones europeas, codecisión, acuerdo en primera lectura, conciliación, trílogos.

Abstract: The purpose of the article is to analyse the functioning of the ordinary legislative procedure $(O L P)$ in practical terms and how the practice of «trilogues» and first reading agreements is increasingly consolidated. The description of the OLP in Article 294 TFEU does not, in itself, give an accurate picture of the applied functioning of the OLP. One should consider the Joint Declaration of the

* Recibido el 16 de diciembre de 2014, aceptado el 19 de enero de 2015.

** Letrada del Parlamento Europeo. Este trabajo ha sido realizado a título personal y las opiniones expresadas no vinculan en modo alguno a la Institución en la que la autora tiene el honor de prestar sus servicios. 
European Parliament, the Council and the Commission on practical arrangements for the codecision procedure. These arrangements entail that the number of legislative acts adopted at first reading is constantly increasing, while the number of legislative files arriving at the conciliation phase has decreased significantly. The role of the rapporteur and the competent parliamentary committee as well as the role of the six-month Council Presidency are reinforced, in that they have become the negotiators on behalf of their institution.

Keywords: Ordinary legislative procedure (OLP), European institutions, codecision, first reading agreement, conciliation, trilogue.

\section{Introducción}

La evolución del procedimiento de codecisión (actualmente procedimiento legislativo ordinario) desde sus inicios hasta ahora, ha venido marcada por una clara tendencia a adoptar los actos legislativos en las primeras fases del procedimiento y a limitar en todo lo posible que los procedimientos lleguen a la tercera lectura o fase de conciliación. Lo que se empezó haciendo de forma informal, mediante contactos entre la presidencia de turno del Consejo y los ponentes del Parlamento Europeo con el fin de llegar a un acuerdo informal en primera lectura, se ha venido afianzando con los años y, sobre todo, ha perdido el carácter espontáneo e informal que tenía en sus inicios. Así, actualmente, estos contactos en primera lectura han quedado enmarcados en una seria de reglas que rigen todo un proceso de negociación y conclusión de acuerdos entre los dos co-legisladores.

\section{La evolución de la codecisión: los acuerdos en primera lectura}

Desde la introducción en el Tratado de Maastricht del procedimiento legislativo conocido como «codecisión» hasta hoy, veinte años después, los distintos Tratados han ido ampliando cada vez más el campo de aplicación de la codecisión. Hoy día la mayoría de las bases jurídicas del TFUE reenvían al procedimiento de codecisión, llamado ahora procedimiento legislativo ordinario (PLO). Por un lado, sectores que antes no estaban cubiertos por la codecisión (como la política agrícola, la política de pesca, la política comercial común o el antiguo «tercer pilar»), están sujetos desde el TFUE al PLO, y, por otro lado, nuevas bases jurídicas inexistentes hasta ahora (energía, turismo, deporte) habilitan al legislador de la Unión a adoptar disposiciones también según el PLO. El PLO se convierte así en el procedimiento legislativo más utilizado por el legislador de la Unión. De hecho, 
en la legislatura 2009-2014, el 89\% de las propuestas legislativas presentadas por la Comisión se corresponden con el PLO y sólo el $11 \%$ son propuestas para ser adoptadas mediante el procedimiento legislativo especial ${ }^{1}$. Ello contrasta con los datos de la legislatura anterior (2004-2009) en la que el $49 \%$ de las propuestas legislativas de la Comisión lo eran en codecisión. Sin duda el traspaso de políticas europeas «clásicas» hacia la codecisión (agricultura, pesca, política comercial), así como el de todas las disposiciones en materia de asilo, inmigración, justicia, explican este aumento de la legislación adoptada por el PLO, más que la introducción de bases jurídicas completamente nuevas en ámbitos en los que hasta ahora el legislador de la UE carecía de competencias, cuya contribución cuantitativa al conjunto de normas aprobadas por PLO no es muy importante ${ }^{2}$.

$\mathrm{Al}$ analizar el papel de las instituciones en el procedimiento legislativo, lo primero que se aprecia es que la ampliación de los sectores cubiertos por el PLO supone, evidentemente, un aumento de poder del Parlamento que, con el transcurso de los años, se ha convertido en co-legislador en pie de igualdad con el Consejo. En lo que respecta al procedimiento legislativo propiamente dicho y sus diferentes lecturas, no se puede decir que el TFUE haya modificado el papel de cada institución (Parlamento, Consejo, Comisión) durante el procedimiento de adopción de los actos legislativos. El artículo 294 TFUE no supone un cambio en el papel de las instituciones con relación al que tenían según el artículo $251 \mathrm{CE}^{3}$. Lo cierto es que para estudiar el papel de las instituciones en el PLO, la lectura del artículo 294 TFUE, y de su predecesor el artículo 251CE, no nos da muchas pistas. Para ver el papel real que desempeña cada institución hay que detenerse en las reglas relativas a las modalidades prácticas de la codecisión de las que se han dotado las instituciones y, cómo no, a su aplicación en la práctica.

El PLO, tal como lo prevén los Tratados, consta de tres lecturas, siendo la tercera lectura la fase de negociación o de «conciliación» entre Parlamento y Consejo, cuando no se ha logrado la adopción del acto legislativo ni en primera ni en segunda lectura. La única instancia de negociación en-

${ }^{1}$ Las cifras y datos que se citan a lo largo de este capítulo proceden de la Dirección general de políticas internas (DG IPOL) del Parlamento europeo.

2 En la legislatura 2009-2014 ha habido 488 actos legislativos adoptados por el PLO, de los que 201 corresponden a un PLO introducido en el Tratado de Lisboa y 27 corresponden a nuevas bases jurídicas del Tratado de Lisboa.

${ }^{3}$ El mayor cambio institucional en el procedimiento legislativo de codecisión post-Lisboa afecta a los Parlamentos nacionales y a sus nuevos poderes de control de la subsidiariedad en virtud de los Protocolos 1 (sobre el cometido de los Parlamentos nacionales en la Unión europea) y 2 (sobre la aplicación de los principios de subsidiariedad y proporcionalidad), más que a las propias instituciones europeas cuyo papel en el artículo 294 TFUE viene a ser el mismo que tenían bajo el régimen del artículo $251 \mathrm{CE}$. 
tre representantes del Parlamento y del Consejo que prevé el PLO descrito en el artículo 294 TFUE es el «comité de conciliación» compuesto por los miembros del Consejo y un número igual de representantes del Parlamento (es decir, 28 diputados) que deben intentar llegar a un acuerdo sobre un texto conjunto basado en las posiciones del Parlamento Europeo y del Consejo en segunda lectura.

En la práctica, en la fase de tercera lectura, se han venido desarrollando desde los inicios de la codecisión, negociaciones informales preparatorias de la reunión formal del comité de conciliación: los llamados «trílogos» informales en los que un equipo negociador por parte del Parlamento (no todos los diputados que forman parte del comité de conciliación), un representante de la Presidencia del Consejo (es decir, no los representantes de los Estados miembros en el Consejo, sino sólo la Presidencia) y representantes de la Comisión, se reúnen para ir avanzando en la negociación en reuniones más restringidas e informales que las reuniones del comité de conciliación. Después de cada trílogo, los negociadores rinden cuenta de los resultados cada uno en su institución - los negociadores del Parlamento ante la Delegación compuesta actualmente de 28 diputados y la Presidencia del Consejo ante el Consejo - y reciben allí el aval o no a lo ya negociado así como instrucciones sobre la estrategia de negociación a seguir en el siguiente trílogo. Así trílogo a trílogo, en estos diálogos tripartitos o a tres bandas en «petit comité» se va llegando a un texto conjunto que, una vez aprobado por el comité de conciliación, se someterá a la aprobación del Consejo y a la aprobación del Parlamento en pleno.

En realidad, el comité de conciliación no lleva a cabo un verdadero debate sobre el texto ni un análisis detallado (artículo por artículo y considerando por considerando). Actualmente es imposible que las reuniones del comité de conciliación, en las que se reúnen representantes de los 28 Estados miembros, más los 28 diputados de la delegación del Parlamento, más los representantes de la Comisión, todos ellos acompañados del personal técnico correspondiente (con lo que se llega fácilmente a más de cien participantes), sean reuniones de trabajo o de negociación y debate, sino que se convierten en reuniones formales que consisten en aprobar (o en rechazar) el texto conjunto que ha sido negociado por los negociadores del Parlamento y la presidencia del Consejo durante los trílogos.

Así pues, en la fase de conciliación las relaciones entre las instituciones y la forma de trabajar los textos legislativos es completamente distinta a la dinámica de las primeras y segundas lecturas tal y como las concibe el TFUE. En conciliación, los representantes de los dos co-legisladores trabajan sobre el texto de manera conjunta durante los trílogos, mediante contactos y negociaciones directas, en lugar de examinar cada uno la propuesta de la Comisión de forma unilateral dentro de su institución y de aprobar 
«su» posición. De lo que se trata es de llegar a un texto común resultado del compromiso alcanzado tras las negociaciones.

Durante la fase de conciliación el papel de la Comisión es también completamente diferente al que tiene en primera y segunda lectura. Su papel, tal y como lo establece de forma explícita el artículo 294 TFUE, es el de participar en los trabajos y tomar todas las iniciativas necesarias para propiciar un acercamiento entre las posiciones del Parlamento y del Consejo. Para ello, puede, obviamente, proponer fórmulas de compromiso sobre tal o cual aspecto controvertido, y puede manifestar su posición sobre los acuerdos parciales a los que van llegando los negociadores del Parlamento y del Consejo durante los trílogos, así como sobre el resultado final. Sin embargo, su oposición a las soluciones a las que lleguen los co-legisladores no condiciona ni impide la adopción del acto legislativo: la Comisión no puede, en esta fase del procedimiento, ni modificar, ni retirar su propuesta legislativa; los co-legisladores pueden adoptar el texto común que se convertirá en el acto legislativo, aun cuando cuenten con la oposición de la Comisión ${ }^{4}$. Sí que puede, claro está, manifestar dicha oposición y, si así lo decidiere, recurrir el acto legislativo adoptado por los co-legisladores ante el Tribunal de Justicia 5 .

Si hemos explicado esta dinámica negociadora de la fase de conciliación y la importancia de los trílogos informales, no previstos en el artículo 294 TFUE, es porque sin comprender bien este mecanismo de la conciliación no se puede comprender el funcionamiento actual de todo el PLO, primera y segunda lectura incluidas.

De hecho, desde el Tratado de Ámsterdam el procedimiento de codecisión ha venido evolucionando de tal manera que el formato «conciliación» - que se basa, como hemos visto, en el diálogo interinstitucional permanente, mediante trílogos informales en los que se negocian los textos legislativos entre representantes de las distintas instituciones - se ha extendido a las primeras fases del procedimiento. Las tres instituciones han optado por la vía de los acuerdos negociados, ya desde la primera lectura, lo que ha cambiado los métodos de trabajo y las relaciones entre instituciones durante

${ }^{4}$ En contraste, en primera y en segunda lectura, la oposición de la Comisión desencadena la necesidad de voto por unanimidad en el Consejo sobre las enmiendas objeto de un dictamen negativo por parte de la Comisión.

5 Ver por ejemplo, asunto C-378/00 Comisión / Parlamento y Consejo ( $(L I F E »)$, EU:C:2003:42, o el asunto C-122/04 Comisión / Parlamento y Consejo ( Forest Focus»), EU:C:2006:134. En los dos casos el desacuerdo entre la Comisión, de un lado, y el Parlamento y el Consejo, del otro, versaba sobre las disposiciones «comitología» decididas por los co-legisladores en conciliación con la oposición de la Comisión. En el primer caso, el Tribunal anuló las disposiciones impugnadas por la Comisión; en el segundo, el Tribunal desestimó el recurso de la Comisión. 
la primera y segunda lectura, y ha reducido considerablemente el número de actos legislativos que acaban llegando a la fase de conciliación.

Esta nueva óptica se refleja en la «Declaración común sobre las modalidades prácticas del nuevo procedimiento de codecisión (artículo 251 del Tratado constitutivo de la Comunidad europea)» de $1999^{6}$ en la que las tres instituciones ponen de manifiesto su voluntad de cooperar «de buena fe con objeto de acercar al máximo sus posiciones de modo que, en la medida de los posible, el acto pueda ser adoptado en primera lectura» y se comprometen a establecer «los contactos apropiados para realizar un seguimiento de la evolución de los trabajos y analizar su grado de convergencia.» En caso de que el procedimiento legislativo llegue a la fase de segunda lectura, las tres instituciones afirman que «se podrán establecer los contactos apropiados con objeto de comprender mejor las posiciones respectivas y permitir una conclusión lo más rápida posible del procedimiento legislativo.» Es decir, sin entrar en detalles sobre en qué ha de consistir dicha «cooperación de buena fe», ni tales «contactos apropiados», lo que está claro ya desde el Tratado de Ámsterdam es que las tres instituciones han resuelto evitar en lo posible que los procedimientos legislativos se dilaten en el tiempo y acaben llegando a la tercera lectura o fase de conciliación.

¿En qué consisten estos «contactos apropiados»? ¿Cuál es el papel de cada institución en estos acuerdos en primera lectura? Lo que se ha hecho en la práctica ha sido trasladar el mecanismo de las conciliaciones (pero sin comité de conciliación) a la primera lectura. Así, cuando el TFUE dice en su artículo 294 apartado 3 que «el Parlamento europeo aprobará su posición de primera lectura y la transmitirá al Consejo», ello significa en la práctica que representantes del Parlamento negocian con la presidencia de turno del Consejo, y con la participación de la Comisión (es decir en «trílogo»), las enmiendas que conformarán la posición del Parlamento, que, por tanto, no será en realidad la posición unilateral del Parlamento, sino que será una posición ya negociada con la presidencia del Consejo. De tal forma, que cuando dichas enmiendas de primera lectura «negociadas» sean aprobadas por el pleno del Parlamento, éste sabe que cuenta ya de antemano con el compromiso del Consejo de aprobar la posición del Parlamento tal cual, de forma que el acto legislativo será adoptado en primera lectura ${ }^{7}$.

${ }^{6}$ DO C 148 de 28 de mayo de 1999, p.1.

${ }^{7} \mathrm{El}$ acuerdo puede darse también en otras etapas del procedimiento. Si no ha habido acuerdo en primera lectura del Parlamento, el acuerdo puede consistir en negociar la posición del Consejo con el Parlamento, de forma que el Consejo sabe al aprobar su posición que el Parlamento la aceptará tal cual (ya que no será en realidad «su» posición sino una posición negociada con el Parlamento) y que no habrá lugar a una segunda lectura. Esto es lo que se conoce como un «early second reading agreement». El acuerdo puede también tener por ob- 
Estas negociaciones en trílogos informales, han sido objeto de críticas, principalmente en cuanto a la falta de transparencia, y han generado cierto malestar dentro de las instituciones, especialmente en el Parlamento europeo. Ello porque un número reducido de negociadores se convierte en interlocutor con la otra institución, con la que llega a acuerdos, que luego la institución en nombre de la que han negociado se ve más o menos compelida a aceptar si no quiere cargar con la responsabilidad de la ruptura del acuerdo y del fracaso de la negociación.

Por todo ello, desde la Declaración común sobre las modalidades prácticas del nuevo procedimiento de codecisión de 1999, antes mencionada, hasta ahora, se han ido detallando una serie de reglas y de medidas que regulan el mandato de negociación y la rendición de cuentas por parte de los negociadores. El objetivo es mejorar la transparencia, la pluralidad y la participación de todos los grupos políticos en el debate, sin que ello afecte a la rapidez y a la eficacia de las negociaciones. Es decir, que aunque sigue tratándose de trílogos informales, ya que ni su existencia misma, ni su composición, estructura, contenido o frecuencia están establecidos en los Tratados, sí se les ha dotado de un cierto marco regulador (más en el caso del Parlamento que en el caso del Consejo), sobre todo en lo que respecta al mandato de negociación y a la formalización de los acuerdos, mientras que el desarrollo en sí de las negociaciones sigue siendo informal. Es decir, trílogos informales sí, pero sin que eso signifique, ni mucho menos, que los negociadores actúen «por libre» sino que lo hacen en ejecución de un mandato claramente establecido (principalmente en el caso del Parlamento) y sometidos a una obligación de información y rendición de cuentas.

Ya las tres instituciones convinieron en la necesidad de encarrilar estos trílogos informales y decidieron en 2007 actualizar y detallar la Declaración común del Parlamento Europeo, del Consejo y de la Comisión sobre las modalidades prácticas del procedimiento de codecisión (artículo 251 del Tratado CE) estableciendo la necesidad de un mandato de negociación claro $^{8}$. En dicha declaración se establece que «en general, estos diálogos tripartitos se celebran en un marco informal. Pueden celebrarse en todas las fases del procedimiento y con diferentes niveles de representación, según la naturaleza del debate de que se trate. Cada institución, de conformi-

jeto las enmiendas del Parlamento en segunda lectura, en caso de que se haya llegado a esta fase del procedimiento. Por consiguiente, en la práctica, el Consejo adoptará «su» posición de primera lectura, cuando no haya sido posible llegar a un acuerdo sobre las enmiendas del Parlamento en primera lectura; la segunda lectura sólo tendrá lugar cuando no haya sido posible un acuerdo sobre la posición de primera lectura del Consejo. Así, la conciliación se concibe como la consecuencia del fracaso de las negociaciones en primera y segunda lectura.

${ }^{8}$ DO C 145 de 30 de junio de 2007, p.5. 
dad con su reglamento interno, designará a sus representantes para cada reunión, definirá su mandato e informará con antelación suficiente a las otras instituciones acerca de las modalidades de las reuniones.»

El Parlamento europeo aprobó el 20 de noviembre de 2012 una reforma de su Reglamento interno con el fin precisamente de establecer estas reglas, a las que hace referencia la Declaración común, sobre la apertura, el mandato y el desarrollo de las negociaciones con el Consejo ${ }^{9}$.

Estos acuerdos en primera lectura constituyen una práctica ya bien rodada, que procede como hemos visto de la aplicación del Tratado de Ámsterdam y que no hace sino reforzarse y asentarse con el paso del tiempo. Ello ha supuesto - con relación a las primeras lecturas no negociadasun cambio sustancial en la manera en que cada institución examina la propuesta de la Comisión. En el Parlamento, ya no se trata solamente de lograr la mayoría necesaria para la aprobación de las enmiendas o de lograr un compromiso entre grupos políticos para que las enmiendas tengan el mayor apoyo posible dentro del Parlamento. Se trata de, al mismo tiempo, llegar a un texto de compromiso con el Consejo. Igualmente, el Consejo se tiene que preocupar no sólo de lograr el necesario equilibro entre las posturas de los distintos Estados miembros para lograr la mayoría necesaria (o en su caso, la unanimidad), sino también de que el resultado de dichos equilibrios nacionales sea a su vez aceptable para el Parlamento. De esta forma, se desarrollan de forma paralela intensas negociaciones dentro de cada institución y entre instituciones ${ }^{10}$.

\section{La apertura de las negociaciones: el mandato de negociación}

Todo procedimiento legislativo ordinario comienza cuando la Comisión aprueba su propuesta legislativa ${ }^{11}$ y la envía al Parlamento Europeo y al

9 Ver los artículos 73 y 74 del Reglamento del Parlamento Europeo, así como el Código de conducta para la negociación en el marco del procedimiento legislativo ordinario (aprobado por la Conferencia de presidentes de los grupos políticos en su reunión de 18 de septiembre de 2008) que figura como anexo XX del Reglamento del Parlamento. http://www.europarl.europa.eu/sides/getLastRules.do?language $=\mathrm{ES} \&$ reference $=\mathrm{TOC}$

${ }^{10}$ El número de trílogos que se celebran está en constante aumento. En la legislatura 2009-2014 ha habido más de mil trílogos sobre unas 350 propuestas legislativas en PLO. La comisión de asuntos económicos y monetarios es la que más trílogos ha celebrado en este periodo (331) seguida de la comisión de medio ambiente (172).

11 El Tratado de Lisboa mantiene - en gran medida - en la Comisión el monopolio de la iniciativa legislativa; salvo excepciones (como por ejemplo los artículos 129 p. 4, 223 p. 2, 228 p. 4, 257 o 281 TFUE) los co-legisladores tienen encima de la mesa como texto de base, una propuesta de la Comisión. 
Consejo para su adopción. En el Parlamento, la propuesta se atribuye a una comisión parlamentaria ${ }^{12}$.

Según la última modificación del Reglamento del Parlamento Europeo, corresponde a la comisión parlamentaria competente decidir sobre la oportunidad de abrir negociaciones con la presidencia de turno del Consejo, con vistas a un acuerdo en primera lectura, o si es preferible proceder a la adopción de una primera lectura del Parlamento en la que se recojan únicamente las enmiendas que el Parlamento apruebe de forma unilateral sin establecer contactos con el Consejo. Es decir, que la comisión competente identifica, caso por caso, las propuestas candidatas a poder ser objeto de un acuerdo en primera lectura y aquellas que no parecen prestarse a tales acuerdos. Esta decisión se adopta por votación y necesita un voto por mayoría de los miembros que componen la comisión ${ }^{13}$. La decisión se comunica al Presidente del Parlamento quien a su vez informa a la Conferencia de presidentes de los grupos políticos, con lo que, la comisión competente no actúa de forma aislada, sino que los grupos políticos están al tanto de la apertura de las negociaciones. De esta forma, el ponente responsable de la propuesta legislativa de que se trate, o el presidente de la comisión competente, no pueden iniciar negociaciones con la Presidencia del Consejo si no ha habido previamente una decisión en este sentido aprobada por la mayoría de los miembros de la comisión competente.

Una vez decidido que se va a intentar negociar un acuerdo de primera lectura, la comisión competente nombra al equipo negociador compuesto por el ponente, el presidente de la comisión competente (que pueden ser o no del mismo grupo político), así como los llamados ponentes ficticios o ponentes alternativos («shadows»), es decir los ponentes designados por los demás grupos políticos, de tal forma que un representante de cada grupo político participa en las negociaciones.

Antes de empezar las negociaciones, la comisión competente debate y aprueba (por mayoría simple) su informe con las enmiendas que se propongan. Dicho informe constituye el mandato de negociación para el equipo negociador del Parlamento ${ }^{14}$. Es decir, que no será este informe el que se someta tal cual al voto del pleno (como ocurriría si se hubiera de-

12 Según la distribución de competencias entre comisiones que se recoge en el anexo VI del Reglamento del Parlamento.

13 Artículo 73 del Reglamento del Parlamento Europeo.

14 El Reglamento del Parlamento también prevé la posibilidad, con carácter excepcional (artículo 74), de iniciar las negociaciones sin haber votado previamente un informe con enmiendas en la comisión competente. En este caso, la decisión de abrir negociaciones se somete a la Conferencia de presidentes de los grupos políticos, que puede decidir su inclusión en el orden del día de un pleno; el mandato de negociación constará de orientaciones, objetivos, o prioridades que no toman la forma de informe votado en la comisión competente. 
cidido no proceder a un acuerdo con el Consejo en primera lectura), sino que este informe sirve de base al equipo negociador del Parlamento para llevar a cabo las negociaciones. Será el resultado de estas negociaciones (en el que algo del informe inicial se habrá tenido que ceder y al que se habrán incorporado algunas aspiraciones del Consejo) el que se someta al voto del pleno.

Lógicamente, la negociación es cosa de dos, y no basta con la voluntad de la comisión parlamentaria — por mucha mayoría absoluta con la que haya sido adoptada - de negociar un acuerdo en primera lectura para que la negociación tenga lugar. El Consejo debe estar también en disposición de iniciar negociaciones sobre la propuesta legislativa en cuestión. Normalmente, el programa de cada Presidencia indica cuáles son sus prioridades y cuáles son las propuestas legislativas sobre las que la Presidencia tiene interés especial en empezar los trílogos.

Por parte del Consejo no existe una obligación legal de disponer de un mandato formal para que el representante de la Presidencia pueda participar en un trílogo, si bien la Presidencia suele solicitar un mandato y en todo caso ha de tenerlo si se va a llegar a un acuerdo con el Parlamento. No obstante, se admite que la Presidencia pueda participar previamente en contactos o conversaciones con el equipo negociador del Parlamento sin necesidad de un mandato formal. Es costumbre que, al principio de cada Presidencia, se organicen reuniones con cada presidente de comisión parlamentaria para pasar revista a las distintas propuestas legislativas pendientes que serán objeto de futuras negociaciones. El mandato de la Presidencia es a veces general, a veces toma la forma de un texto concreto (una «orientación general» $)^{15}$.

\section{Las negociaciones entre Parlamento y Consejo; el papel de la Comisión: los trílogos}

Los trílogos son clave para lograr un acuerdo. De hecho, la verdadera negociación tiene lugar en el trílogo. Los participantes en el trílogo examinan la propuesta de la Comisión al detalle, artículo por artículo y considerando por considerando. Ya hemos visto que, por parte del Parlamento, participan en el trílogo el ponente, el presidente de la comisión competente y los ponentes de los demás grupos políticos asistidos por personal de la se-

15 Ver las declaraciones del entonces Director en el Servicio Jurídico H. Légal a la House of Lords en House of Lords European Union Committee- 17th Report of session 2008-09 "Codecision and national parliamentary scrutiny- Report with evidence" (21 julio 2009-HL Paper 125; página 52 de las «minutes of evidence») 
cretaría de la comisión y del servicio jurídico. Por parte del Consejo, participan un representante de la Presidencia de turno, normalmente el Representante Permanente o el Representante Permanente Adjunto acompañado de personal de la Representación Permanente, así como de la Secretaría General del Consejo, incluido su servicio jurídico. La Comisión está representada por funcionarios de la Dirección General correspondiente; dependiendo de los trílogos de que se trate, asistirán un Jefe de Unidad o un Director, o incluso un Director General.

Por parte del equipo de negociación del Parlamento, el objetivo será que el acuerdo que resulte de los trílogos se aleje lo menos posible del informe que se votó en la comisión y que constituía su mandato de negociación, de forma que pueda ser aceptado sin reticencias por la comisión competente. Del mismo modo, para la Presidencia del Consejo, el objetivo consistirá en que el acuerdo pueda ser aceptado por el Consejo, y que los Estados miembros vean que sus objetivos y reivindicaciones han quedado reflejados en el acuerdo y puedan, por tanto, aceptar el texto de forma definitiva sin necesidad de pasar a una segunda lectura.

Los trílogos constituyen verdaderas negociaciones. Es decir, que cada uno sabe que tendrá que hacer concesiones y deberá preparar su estrategia de negociación y decidir qué es lo que está dispuesto a abandonar, a cambio de qué, y cuáles son los puntos que considera no negociables. Así, los representantes del Parlamento y del Consejo defienden sus posturas y tratan de llegar a puntos de encuentro para dirimir sus divergencias. Por ejemplo, si el Parlamento desea que el campo de aplicación de la norma en cuestión sea más amplio que el defendido por el Consejo, tal desacuerdo puede saldarse con una cláusula de revisión que prevea una extensión del campo de aplicación en el futuro. No es extraño que la divergencia verse sobre la obligatoriedad o no de algunas disposiciones para los Estados miembros, y si éstos «deberán», «deberían»o «podrán» adoptar tal o cual medida ${ }^{16}$. Otros puntos de desacuerdo pueden ser la duración más o menos larga de los períodos transitorios, que permiten a los operadores del sector adaptarse a las nuevas reglas, el alcance de posibles derogaciones, o el plazo de transposición y, muy en particular, los aspectos que no van a quedar cubiertos por el acto legislativo sino que se dejan a la futura adopción por la Comisión de actos delegados o de medidas de ejecución (las disposiciones «comitología» antes del Tratado de Lisboa), cuestión esta última que se convierte muchas veces en el principal punto de fricción.

16 Muchas veces las discusiones giran en torno a las fórmulas «shall», «should» o «may», ya que la mayoría de las veces se negocia en inglés. 
Después de cada trílogo, el equipo negociador del Parlamento informa a la comisión competente del estado de la negociación y se debaten los siguientes pasos a dar en el próximo trílogo. Los miembros de la comisión, que no forman parte del equipo negociador, son así informados de la marcha de las negociaciones y pueden ir actualizando el mandato y las instrucciones de negociación. El equipo negociador actúa así en los trílogos como portavoz de la comisión parlamentaria. Por su parte, la Presidencia del Consejo informa igualmente a las delegaciones nacionales, ya sea en nivel de grupo de trabajo o en Coreper (Comité de Representantes Permanentes de los Gobiernos de los Estados miembros - COREPER -) Como ya hemos visto, una vez que se acerca el momento de concluir el acuerdo, la Presidencia no se puede comprometer a un acuerdo si no ha sido mandatada expresamente para ello por el Coreper ${ }^{17}$.

Durante los trílogos, por parte del Consejo, sólo asiste la Presidencia, mientras que por parte del Parlamento participan el ponente y los ponentes de los demás grupos políticos, es decir, están representados todos los grupos políticos, de tal forma que el ponente está en cierta forma «vigilado» por los demás grupos durante las negociaciones, quienes pueden ver con sus propios ojos si el ponente defiende adecuadamente la posición de negociación acordada y si no «barre para su casa» (para su grupo político). Tienen así una información de primera mano sobre lo que sucede en los trílogos, que no tienen los Estados miembros, quienes tienen que confiar en las informaciones que les facilite la Presidencia.

Durante la primera y segunda lecturas, el papel que el TFUE otorga a la Comisión, es muy distinto al que tiene durante le fase de conciliación. En primera lectura, el artículo 294 TFUE ni siquiera menciona a la Comisión $^{18}$. Los redactores del Tratado no previeron ninguna función especial para la Comisión durante la fase de primera lectura. El apartado 2 del artículo 294 TFUE dice simplemente que la Comisión presenta su propuesta al Parlamento y al Consejo, tras lo cual estas instituciones proceden a su primera lectura sin intervención de la Comisión (apartados 3 a 5). Lo único que tiene que hacer la Comisión es, una vez que el Parlamento ha adoptado su primera lectura, informarle de cuál es su posición sobre las enmiendas adoptadas (apartado 6) ${ }^{19}$. En segunda lectura además de informar de su po-

17 Ver "House of Lords European Union Committee- 17th Report of session 2008-09; Codecision and national parliamentary scrutiny- Report with evidence" antes citado.

18 Artículo 294 TFUE, apartados 3 a 5.

19 De una lectura combinada de los artículos 293 TFUE y 294 TFUE se deduce que si la Comisión se opone a las enmiendas del Parlamento y no las hace suyas integrándolas en su propuesta, entonces será necesaria la unanimidad en el Consejo para poder enmendar la propuesta de la Comisión. 
sición sobre las enmiendas, al igual que en primera lectura (apartado 7.c), su oposición sobre las enmiendas provoca también la unanimidad en el Consejo (apartado 9).

Así pues, en primera y segunda lectura, el Tratado no le confiere a la Comisión la tarea de facilitadora de acuerdos que sí le atribuye de forma explícita en tercera lectura. Durante la primera lectura, el texto sobre el que se negocia es la propuesta de la Comisión, de la que la Comisión es dueña y puede, por consiguiente, modificar o - en principio - incluso retirar. Sin embargo, en los acuerdos en primera, o en segunda, lectura la Comisión tiene un doble papel: el propio de las primeras fases del PLO (autor de la propuesta que manifiesta su posición sobre las enmiendas) pero al mismo tiempo el propio de mediador en una negociación entre co-legisladores con el fin de que éstos lleguen a un texto de compromiso. De hecho, la Declaración común sobre las modalidades prácticas de la codecisión de 2007, estipula que «la Comisión favorecerá los contactos y ejercerá su derecho de iniciativa de manera constructiva con vistas a facilitar un acercamiento de las posiciones del Parlamento Europeo y del Consejo, respetando el equilibrio interinstitucional y el papel que le confiere el Tratado» ${ }^{20}$. Dicho de otra forma, la Comisión tiene como misión acercar posturas entre Parlamento y Consejo, y puede para ello proponer vías de salida cuando vea que las divergencias entre Parlamento y Consejo se encallan, pero sin perder por ello el papel que le atribuye el Tratado. En la práctica, sin embargo la Comisión no ejerce plenamente su papel de primera lectura y se comporta de forma similar a la conciliación. La Comisión utiliza rara vez la posibilidad de provocar el voto por unanimidad en el Consejo, y más raramente todavía recurre a la drástica decisión de retirar su propuesta ${ }^{21}$. Por regla general, se limita - como en la fase de conciliación - a, llegado el caso, manifestar su desacuerdo, pero sin oponerse formalmente y sin desencadenar la necesidad del voto por unanimidad en el Consejo, o, como mucho, a anunciar que si

20 Punto 17 de la Declaración en lo que se refiere a la primera lectura, y punto 22 en lo que respecta a la segunda lectura.

${ }^{21}$ Lo ha hecho, de forma excepcional, en el caso de la propuesta de Reglamento por el que se establecen las disposiciones generales de la ayuda macrofinanciera a terceros países (COM) 2011 0396; 2011/0176(COD). Dada su oposición al acuerdo hacia el que se encaminaban los dos co-legisladores con relación a la supresión de determinadas disposiciones de la propuesta inicial de la Comisión relativas a la adopción ulterior por la Comisión de medidas de ejecución, la Comisión decidió el 28 de mayo de 2013 retirar su propuesta de Reglamento, poniendo así fin al procedimiento legislativo. Esta decisión de la Comisión ha sido recurrida por el Consejo ante el Tribunal de Justicia, de forma que el Tribunal deberá pronunciarse próximamente sobre el derecho de la Comisión a retirar su propuesta durante la fase de primera lectura y sobre si - como pretende el Consejo - existen limitaciones a dicho derecho (asunto C-409/13 Consejo/Comisión; conclusiones del abogado general Jääskinen de 18 de diciembre 2014 EU:C:2014:2470. 
el texto es aprobado ejercerá su derecho de recurso ante el Tribunal de Justicia $^{22}$.

Aunque los negociadores son los representantes de los dos co-legisladores mientras que la Comisión tendría un papel de mediación, no hay que subestimar la influencia de la Comisión en el resultado de las negociaciones. Primero porque lo que se discute es su propuesta, es decir que el texto de base es el texto de la Comisión y lo que se discuten son enmiendas a dicho texto. Así pues, es la Comisión quien ha establecido a priori - en virtud de su poder de iniciativa legislativa - sobre qué se va a legislar, con qué alcance, con qué limitaciones, en qué condiciones etc. y es por tanto la Comisión quien establece el marco en el que se van a desarrollar las negociaciones. Pero también, porque como autora de la propuesta, y de todos los estudios y evaluaciones de impacto previos que le han llevado a adoptar la propuesta, es la Comisión quien dispone de los conocimientos técnicos sobre la materia indispensables para poder decidir sobre la legislación en cuestión. Además, los representantes de la Comisión en los trílogos son funcionarios de la Dirección General correspondiente (es decir, personal técnico, ya que en los trílogos informales de primera lectura no participa el Comisario responsable), mientras que por parte del Consejo y del Parlamento la representación es política. Por ello, la Comisión puede tener gran influencia en el resultado final ya que no es raro que los representantes políticos del Consejo y del Parlamento se dirijan a la Comisión en busca de ayuda sobre tal o cual punto, o que lo que digan los funcionarios de la Comisión sobre la viabilidad de tal o cual propuesta del Consejo o del Parlamento o sobre posibles soluciones alternativas, sea muy tenido en cuenta.

Como vemos, estas negociaciones en primera lectura se asemejan a las de los trílogos informales preparatorios del comité de conciliación en tercera lectura. Sin embargo, no hay que perder de vista que no tienen la misma naturaleza ni, desde luego, las mismas consecuencias. En tercera lectura, si la negociación fracasa y no se llega a un acuerdo en concilación, no habrá entonces adopción del acto legislativo y se pondrá fin de forma definitiva al procedimiento legislativo sin haber obtenido ningún resul-

${ }^{22}$ Por ejemplo, en el caso del Reglamento 528/2012 del Parlamento Europeo y del Consejo de 22 de mayo de 2012 relativo a la comercialización y al uso de los biocidas (DO L 167 de 27 de junio de 2012, p.1) que fue objeto de un acuerdo entre Parlamento y Consejo en segunda lectura. La Comisión hizo una declaración durante la adopción en el pleno del Parlamento para manifestar su desacuerdo con las disposiciones que le conferían ciertos poderes para adoptar medidas de ejecución según lo previsto en el artículo 291 TFUE, en lugar de prever la adopción de actos delegados en virtud del artículo 290 TFUE. Luego, presentó ante el Tribunal un recurso de anulación de dichas disposiciones, que el Tribunal desestimó (asunto C-427/12 Comisión / Parlamento y Consejo, EU:C:2014:170). 
$\operatorname{tado}^{23}$. En cambio, en caso de fracaso de las negociaciones en primera lectura, el procedimiento legislativo «normal» sigue su curso. El Parlamento adoptará su posición de primera lectura, de forma unilateral, que transmitirá al Consejo según lo previsto en el artículo 294, apartado 3, TFUE, y el Consejo - que no estará entonces en condiciones de aceptar tal cual la primera lectura del Parlamento - procederá a adoptar su posición de primera lectura de acuerdo con el artículo 294, apartado 5, TFUE. Se abrirá así paso a la segunda lectura, durante la cual se podrá llegar a la adopción definitiva del acto legislativo.

\section{La formalización del acuerdo}

Hablamos de trílogos informales porque, como hemos visto, no están previstos en el Tratado, ni existen reglas sobre su funcionamiento o duración ${ }^{24}$, más allá de las reglas internas de las que se han dotado las instituciones y, en particular, el Parlamento. Las negociaciones se desarrollan en un clima de diálogo directo y abierto, no sujeto a reglas. Sin embargo una vez logrado el acuerdo, entonces sí que hay una formalización.

Una vez que se ha alcanzado un acuerdo entre el equipo negociador del Parlamento y la Presidencia del Consejo, el acuerdo es sometido por cada parte a sus respectivas instituciones: la comisión competente en el caso del Parlamento, y el Coreper en el caso del Consejo. Dicho acuerdo se formaliza mediante una carta de la Presidencia del Consejo al presidente de la comisión parlamentaria competente, en la cual le indica que si el pleno del Parlamento aprueba en primera lectura las enmiendas resultantes de los trílogos, tal cual, entonces el Consejo se compromete a adoptar definitivamente el acto legislativo en primera lectura. Tras constatar, generalmente en Coreper, que existe la mayoría necesaria para adoptar el texto, es preciso

${ }^{23}$ Como sucedió por ejemplo con la propuesta de Reglamento sobre nuevos alimentos (COM(2007) 0872-2008/0002 (COD) ). En su reunión de 29 de marzo de 2011 el comité de conciliación constató la imposibilidad de llegar a un acuerdo sobre un texto común, debido a las discrepancias sobre las disposiciones relativas a los alimentos derivados de animales clonados y a la falta de acuerdo, una vez más, en cuanto a si la adopción por la Comisión de medidas para el procedimiento de autorización de los nuevos alimentos. debería hacerse mediante medidas de ejecución en virtud del artículo 291 TFUE o mediante actos delegados previstos en el artículo 290 TFUE.

24 Durante la legislatura 2009-2014, la duración media de un procedimiento legislativo terminado con la adopción del acto legislativo en primera lectura ha sido de seis meses. Hay que recordar que el TFUE no impone plazos para la primera lectura, mientras que en segunda y en tercera lectura los co-legisladores se encuentran sometidos a los plazos establecidos por el artículo 294 TFUE para lograr un acuerdo. 
que el Coreper autorice el acuerdo alcanzado por la Presidencia durante los trílogos para que ésta pueda formalizar el acuerdo con el Parlamento y pueda comprometerse en nombre del Consejo.

Por parte del Parlamento, la comisión competente vota también el acuerdo alcanzado, el cual presentará como informe de la comisión competente ante el pleno del Parlamento para su votación. El presidente de la comisión competente se compromete a someter al pleno, de parte de su comisión, las enmiendas resultantes de los trílogos tal cual, y a recomendar que éstas sean adoptadas por el Parlamento en pleno tal y como han sido acordadas con el Consejo. Lógicamente, no puede garantizarle de antemano al Consejo que el pleno votará favorablemente dichas enmiendas (es necesario lograr una mayoría simple en primera lectura), ni tampoco puede garantizar que no se presenten directamente otras enmiendas en el pleno que puedan recibir el apoyo de la mayoría ${ }^{25}$ (de ahí, el condicional de la carta de la Presidencia del Consejo: «si» el Parlamento aprueba el resultado de los trílogos...). Si ello ocurriera, el acuerdo quedaría roto, y el Consejo quedaría liberado de su compromiso de adoptar el acto legislativo en primera lectura. Pero, por regla general, las negociaciones de primera lectura resultan eficaces y se suelen saldar con un acuerdo y con la adopción del acto legislativo en primera lectura. Así, por ejemplo, en la legislatura 2009-2014 el 85\% de los actos legislativos se han adoptado en primera lectura, mientras que sólo un $2 \%$ ha llegado a la fase de conciliación. La evolución hacia los acuerdos de primera lectura es clara: en la legislatura 1999-2004, la proporción fue de $29 \%$ en primera lectura y $22 \%$ en conciliación; en la legislatura 20042009 fue de $72 \%$ en primera lectura y $5 \%$ en conciliación.

No es fácil evaluar si es el Parlamento o el Consejo quien resulta más favorecido por estos acuerdos de primera lectura, ni cuál de las dos instituciones consigue que la otra acepte sus posiciones. En principio, aquel a quien no le importe demasiado llegar hasta la conciliación, en la propuesta legislativa de que se trate, será en principio quien debería encontrarse en situación de fuerza; mientras que aquel que desea evitar la conciliación tendrá tendencia a ser más flexible en sus posiciones, con el fin de lograr el ansiado acuerdo.

Teóricamente, ello parece implicar una cierta desventaja del Consejo, ya que, por regla general, la Presidencia del Consejo parece en principio la

25 En virtud del artículo 169 p.1 del Reglamento del Parlamento, además de la comisión competente, un grupo político o 40 diputados pueden presentar enmiendas directamente en el pleno. El papel de los ponentes ficticios o «shadows» es fundamental durante las negociaciones para representar cabalmente el parecer de los grupos políticos a los que pertenecen, de forma que al final éstos puedan aceptar el acuerdo que se presenta ante el pleno y no sientan la necesidad de presentar en el pleno nuevas enmiendas, que harían fracasar el acuerdo. 
más interesada en que se logre un acuerdo durante el semestre de su Presidencia, y, por tanto, la que tiene más prisa en la adopción del acto legislativo para poder incluirlo entre los éxitos del balance final de su Presidencia. Sin embargo, en la práctica, los negociadores del Parlamento, aunque no condicionados por el plazo semestral ya que cuentan con toda la legislatura a su disposición, también se ven sometidos a presiones políticas para alcanzar un acuerdo en primera lectura, y tratan también de evitar que recaiga sobre ellos la responsabilidad de la no adopción del acto en primera lectura (lógicamente, cuanto más se acerca el final de la legislatura mayor es la presión para concluir un acuerdo). Así, ambas instituciones intentan que el acuerdo no fracase y que el acto legislativo se adopte en primera lectura.

La posición del Parlamento será más fuerte a la hora de negociar cuanto mayor sea el acuerdo entre grupos políticos. Lo mismo sucede con el Consejo: cuanto mayor sea el acuerdo entre Estados miembros, de forma que si la Presidencia puede hablar con una sola voz, mayor será su capacidad negociadora. Sin embargo si existen importantes divisiones entre Estados miembros, o entre grupos políticos en el Parlamento, más endeble será la posición negociadora de los respectivos portavoces de Consejo y del Parlamento.

Como ya hemos dicho, por regla general, las negociaciones se suelen saldar con un acuerdo. Al final, se podrá hablar de éxito de la negociación cuando los dos co-legisladores vean reflejadas sus ambiciones iniciales, y no demasiado diluidas, en el texto de compromiso, y éste cuente con el apoyo de la Comisión.

Como vemos, las modalidades prácticas del PLO acordadas por las tres instituciones han supuesto que, con los años, la tercera lectura vaya cayendo cada vez más en desuso. Los dos co-legisladores tratan ya desde la primera lectura de lograr un acto legislativo adoptado de común acuerdo, de forma que el papel del ponente y de la comisión parlamentaria competente - por parte del Parlamento - y de la Presidencia de turno del Consejo, se ve reforzado con respecto a las primeras lecturas «no negociadas», ya que se convierten en negociadores y portavoces de sus respectivas instituciones. La Comisión, además de su papel de titular de la iniciativa legislativa, asume también desde la primera lectura un papel activo para propiciar un acuerdo entre el Parlamento y el Consejo, papel que el artículo 294 TFUE le reserva para la tercera lectura.

\section{Conclusiones}

La práctica de los acuerdos en primera lectura se caracteriza por una efervescencia y un frenesí de reuniones informales para negociar los pro- 
yectos de texto legislativo, en las que se van negociando distintas redacciones y distintas versiones de cada considerando y de cada artículo de la propuesta de Reglamento o de Directiva de que se trate, hasta llegar a una versión final que concite el acuerdo de los negociadores de los dos co-legisladores.

Sin embargo, en estos tiempos de transparencia, se hacía cada vez más difícil mantener una situación en la que el debate parlamentario se traslada de las reuniones públicas de las comisiones parlamentarias a los trílogos informales en «petit comité». Por ello, las instituciones, y en particular el Parlamento, se han dotado de reglas que enmarcan el inicio, el desarrollo $\mathrm{y}$ el final de las negociaciones, sometiendo a los negociadores a un escrutinio por parte de los miembros de la comisión parlamentaria al completo, y a una obligación de comparecer periódicamente, en reunión pública, para rendir cuentas del desarrollo de las negociaciones y para recibir instrucciones para la siguiente fase de las negociaciones. Asimismo, durante las negociaciones los ponentes de los demás grupos políticos se ven implicados de forma activa para que todo el espectro político del Parlamento se sienta representado en dichas negociaciones.

De esta forma, aunque durante los trílogos, el debate y la discusión siguen siendo francos y abiertos, y no sujetos a reglas específicas, lo cierto es que ya cada vez menos se puede seguir hablando de acuerdos informales en primera lectura, puesto que la decisión de apertura de las negociaciones, el desarrollo de las mismas y la conclusión final del acuerdo, están sometidos a reglas adoptadas para paliar la falta de transparencia, tantas veces criticada. 